\title{
Information available from brief visual presentations using two types of report
}

CARMEN M. LAWRENCE and JOHN ROSS, University of Western Australia, Nedlands, W. A. 6009

The effects of the acoustic and structural similarity of letters on the accuracy of report were studied using two partial report measures. Ss in Group 1 were required to compare two cued letter pairs on an eight-letter array, and to report whether they were the same or different $(C J)$, while Group 2, in addition to $C J$, had also to report the names of the letters comprising the pairs (CR). The data showed that while $C R$ resulted in a decline in accuracy with increasing cue delay, as reported by Sperling, the $C J$ measure showed an increase in accuracy. Structural similarity of the target pairs increased the difficulty of both $C J$ and $C R$ reports over the first 100 msec. An interpretation is offered in terms of a structural trace which does not decay, and in which structural features are "sharpened" and exaggerated to enhance the discriminability of the components.

Sperling (1960) has demonstrated that the accuracy of a report of a selected row from an array of letters decreases with the delay of an auditory poststimulus cue used to indicate the row to be reported. At brief cue delays the accuracy of partial reports is superior to that predicted from immediate full report performance.

Sperling has taken his findings to imply that there exists a large capacity but rapidly fading visual memory, which can be scanned on cue. He assumes, in common with others (Averbach \& Coriell, 1961; Neisser, 1967; Sperling, 1963, 1967), that more durable storage requires a translation of letters into auditory form, letters not so translated being lost from memory (cf. Turvey, 1967). An implication is that auditory characteristics of letter displays should come to exert an increasing control over recall performance, while visual characteristics diminish in importance. Evidence on the effect of acoustic similarity on performance (Conrad, 1964) is cited in support of a scan-rehearsal model (Sperling, 1967).

The study reported here is designed to compare the effect of structural and acoustic similarity on two types of partial report. One type of report is a content report (CR) of the four letters in a cued row or column of an array. The other type of a report is a comparative judgment $(\mathrm{CJ})$ about the two halves of a cued row or column, Ss reporting whether the halves are the same or different. The study employs a visual poststimulus cue to indicate the target row or column selected for report, since, if visual masking and other interference effects can be avoided, a visual cue should introduce fewer complications than an auditory cue.

\section{Subjects}

Ten volunteer Ss were used, all being students at the University of Western Australia. They were divided into two groups; Group 1 consisting of one male and four females, Group 2 of three males and two females. All Ss had normal or corrected-to-normal visual acuity.

Apparatus

Stimuli were presented in a three-field tachistoscope (Scientific Prototype Model GB) containing a screen 7 in. wide and 5 in. high. The screen was viewed from a distance of 48 in., subtending an angle of $5 \mathrm{deg} 58 \mathrm{~min}$ vertically and $8 \mathrm{deg} 30 \mathrm{~min}$ horizontally. The first channel contained an illuminated fixation cross in the center of a dark adapting field which was displayed continuously. The stimulus arrays and cue display cards were inserted in to Channels 2 and 3 , respectively. Initiation of the experimental sequence was by means of a S-controlled microswitch which triggered the stimulus after a delay of $100 \mathrm{msec}$.

Stimulus Materials

Eight black upper-case letters $1 / 2$ in. high and $1 / 4$ in. wide were printed on to white display cards, each set of eight letters being arranged into four pairs to form two columns and two rows in a rectangular matrix. Letter pairs were spaced 2 deg of visual angle from each other and from the contours of the screen, and all pairs were positioned at least $1 \mathrm{deg}$ from the central fixation point. Component letters of a given pair were separated by $1 \mathrm{deg}$. These precautions were taken to minimize possible contour masking effects. The luminance of the display was $40 \mathrm{ft}-\mathrm{L}$. Strips $1 / 4 \mathrm{in}$. wide and $1 / 2$ in. long were cut from the edges of four black display cards for each of the cue positions-top or bottom row (at the left of the array), and left or right column (at the top of the array). In each case, the card was illuminated from behind and a red filter was placed in front of the visual display. The result was a red bar marker which could be projected onto the viewing screen to indicate a target row or column of the letter display. The contours of the bar marker were always more than 1 deg from the nearest pair.

Construction of the Stimulus Matrices

For some letters of the alphabet it is possible to derive sets of letters which fall into one of four categories in relation to their contrast with the selected letter: (1) acoustically similar-A, (2) structurally similar-S, (3) acoustically and structurally similar-AS, and (4) acoustically and structurally distinct $-D$. Acoustic similarity was defined, in the present study, by the possession of a common phoneme, letters from only two phonemic groups being considered ( $\overline{\mathrm{e}}$ and $\breve{\mathrm{e}})$. Conrad (1964) has shown that in both perceptual and memory tasks, the number of confusions which occur within these groups is greater than the number occurring between them. A list of critical features drawn up by Gibson, Osser, Shiff, \& Smith (1963) was used to give an estimate of the similarity of letters in terms of their structure. Letter pairs were defined as structurally similar if they shared more than $50 \%$ of their features. Confusions between letters can be fairly wet predicted from a measure of their critical feature similarity (Gibson et al, 1963; Gibson, 1968). Letters from the Categories A, S, and D were used in the present experiment, items from each of these categories appearing with equal frequency. In devising the target items for a particular category, a pair of letters was first selected from the group of letters comprising that category. Each component letter of the target pair was then selected from the set of letters appropriately similar or dissimilar to the corresponding letter of the first pair. One hundred twenty such pairs $(N)$ were constructed (40 in each category-A, S, D), these items comprising one half of the stimulus population. The other half of the comparison pairs were identical (I).

In all cases the remaining four letters on the stimulus card were selected randomly from the alphabet, with the restriction that no letter was to be the same as those comprising the target pairs. Letter pairs which formed meaningful words, such as TO or OR, were discarded, and no combination of letters was used twice.

Procedure

Twelve experimental sessions were run for each $\mathrm{S}$. As far as possible, one session was given each day. In each session 120 stimulus and cue cards were presented, identical and different target pairs occurring with equal frequency. The particular set of letters used was redetermined for each new experimental session. The order of the occurrence of stimulus arrays containing identical and different test pairs was randomly decided, and within the latter set, the sequence of cards from $A, S$, and $D$ categories was also randomly determined. Items from each of these three categories occurred with equal frequency. The sequence of the positions of the target pairs was selected at random. Finally, the order of the five cue delays was randomly chosen, with the restriction that each delay occur equally of ten with stimuli containing identical and different test pairs, and within the latter class with equal frequency in each of the categories $A, S$, and D.

Two practice periods were given prior to experimentation to determine the exposure duration at which each observer made $30 \%$ errors when judging identical letter pairs. Following a period of dark adaption, $S$ was read the instructions appropriate to his group, and a number of practice trials given to ensure that he understood the instructions. As soon as he was ready, $\mathrm{S}$ pressed a microswitch which initiated the display sequence. The array of letters was exposed for a duration varying from $S$ to $S$, as outlined above. At one of five predetermined delays after the letter display was turned off $(0,100,250,700,1000 \mathrm{msec})$, the red bar marker was briefly exposed (for the same duration as the letter array), indicating the row or column of the previous display which contained the test pairs. Ss in Group 1 had merely to report whether the indicated letter pairs were the same or 


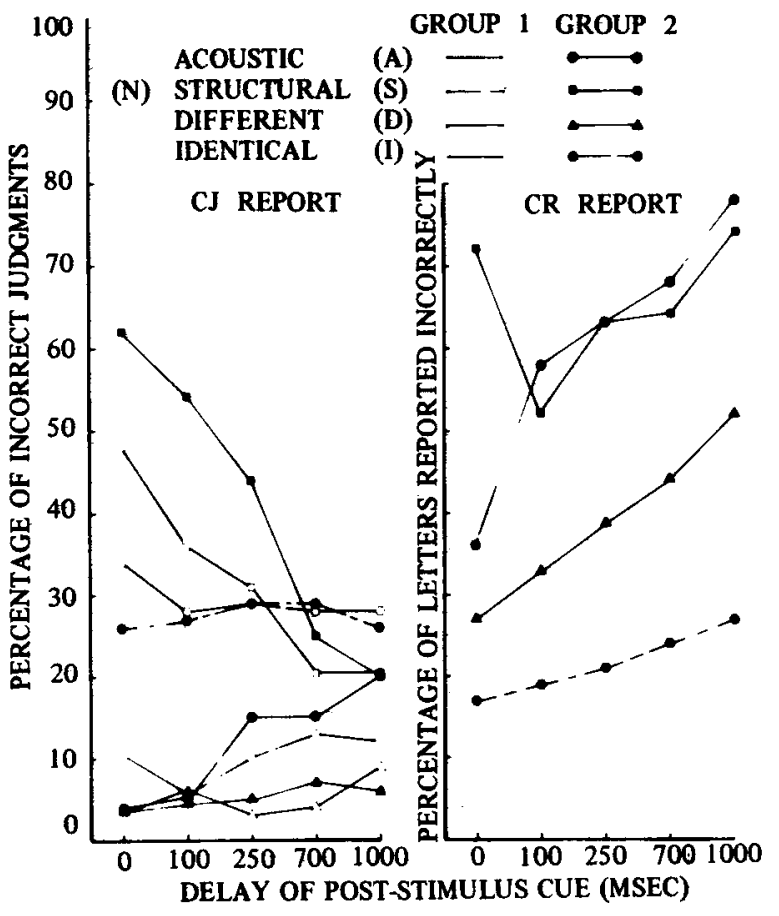

Fig. 19

Fis $\mathbf{~ I b}$

Fig. 1. (a) Percentage of errors in $\mathrm{CJ}$ report for each contrast condition plotted against cue delay; (b) percentage of errors in report of individual letters (CR) in each contrast condition, plotted against cue delay.

different (CJ), while Ss in Group 2 had, in addition, to report the names of the letters (CJ plus CR). Ss in Group 2 were encouraged to adopt their own strategies in relation to the order of reporting $C J$ and $C R$. Between each experimental session, the stimulus exposure duration was adjusted to maintain the level of errors, which the $S$ made in judging identical pairs, at $30 \%$. This was undertaken in an effort to maintain a constant error level in this condition, so that any absolute differences between the other stimulus categories could be determined independently of practice effects and intersubject differences. No adjustment to exposure duration was made within experimental sessions.

\section{RESULTS AND DISCUSSION}

The results are presented in Fig. la which shows CJ results for Group 1 and Group 2, and Fig. Ib which shows CR results for Group 2. Each point in Fig. 1 a represents the percentage of wrong judgments made in one contrast condition $(A, S, D$, or I) at a specified cue delay. Each point in Fig. $1 \mathrm{~b}$ represents the percentage of errors in the reporting of individual letters.

While the CR results show an average decline in accuracy of report with increasing cue delay, as Sperling (1960) found, the CJ results show a trend in the opposite direction both for Group 1, which gave only CJ reports, and for Group 2, which gave both $C J$ and $C R$ reports. Consistency across Ss is striking for both types of report, and it is notable that $\mathrm{CJ}$ curves for each type of contrast are similar for both groups.

The effects of cue delay, of contrast condition and of the interaction of cue delay and contrast are all significant ( $p<.01$ in all cases) for $C J$ reports by Groups 1 and 2 and for CR reports by Group 2. The uniformity of the individual results underlines the clear-cut nature of the effect of contrast, and of the difference in the effect of cue delay on accuracy for different types of contrast.
The two types of report differ not only in that accuracy for CR declines on average where accuracy for $C \boldsymbol{J}$ increases with cue delay, but also in that contrast affects accuracy differently for each type of report. Errors for arrays containing A contrast pairs are low for $C J$ and relatively stable with cue delay, while errors for arrays containing $S$ contrast pairs are high, showing a sharp decline with cue delay. For CR, however, errors for $A$ contrast are high and uniformly increasing with cue delay, whereas errors for $S$ contrast, though also high and generally increasing with cue delay, show an initial decline over the first $100 \mathrm{msec}$. This decline is clearly evident in the results for each $\mathrm{S}$.

The level of errors for I and $\mathbf{N}(A, S, D)$ categories also differs for each report measure. For $C J$, in both groups, the percentage of errors made in judging identical pairs is higher than the percentage of errors made in judging dissimilar pairs (I-30\% errors, $\mathrm{N}-15 \%$ errors). On the other hand, CR measures show that letters comprising identical pairs are more accurately reported than those in which pairs are different (I-20\% errors, $\mathrm{N}-54 \%$ errors).

The difference between $\mathrm{I}$ and $\mathrm{N}$ for $\mathrm{CJ}$ report may at least be partly due to the $O$ adopting a more stringent criterion for making the judgment "same," so that he will more frequently call identical pairs "different" than he will call different pairs "the same," and hence makes more errors for I pairs.

The finding that response accuracy increases when a report measure is used which does not necessitate naming the letters (CJ), argues against an interpretation in terms of a rapidly fading visual trace. The decline in response accuracy reported in the Sperling situation may be a function of the particular response measure which he used (CR). Sperling's $(1963,1967)$ scan-rehearsal model fails to provide an explanation for the findings of this study.

A possible explanation is that the stimulus information can be maintained in a structural form for durations at least as long as $1 \mathrm{sec}$ (cf. Posner, 1967). Components of the array are somehow "sharpened" and exaggerated so that the $O$ is better able to make discriminative judgments about them.

By assuming that the mechanisms which sharpen and exaggerate the structural features of the trace do so at the expense of the detailed information which is necessary to identify each letter, it is possible to explain the increase in error level when the task requires that the $S$ name the letters. The longer the stimulus trace is maintained, the more detailed information is sacrificed, so that at longer delays the identification of letters becomes very difficult.

\section{REFERENCES}

AVERBACH, E., \& CORIELL, A. S. Short term memory in vision. Bell System Technical Journal, 1961, 40, 309-328.

CONRAD, R. Acoustic confusions in immediate memory. British Journal of Psychology, 1964, 55, 75-83.

GIBSON, E. J., OSSER, H., SHIFF, W., \& SMITH, J. An analysis of critical features of letters, tested by a confusion matrix. Final report on Cooperative Research Project No. 639, Office of Education, Department of Health, Education and Welfare.

GIBSON, E. J. Personal communication, 1968.

NEISSER, U. Cognitive psychology. New York: Appleton-CenturyCrofts, 1967.

POSNER, M. I. Short term mernory systems in human information processing. Acta Psychologica, 1967, 27, 267-284.

SPERLING, G. The information available in brief visual presentations. Psychological Monographs, 1960, 74, No. 11.

SPERLING, G. A model for visual memory tasks. Human Factors, 1963 , $5,19-31$.

SPERLING, G. Successive approximations to a model for short-term memory. Acta Psychologica, 1967, 27, 285-292.

TURVEY, M. T. Repetition and the preperceptual information store. Journal of Experimental Psychology, 1967, 74, 289-293. 